\title{
Discourse in Early Alzheimer's Disease Versus Normal Advanced Aging
}

\author{
Sandra Bond Chapman \\ Hanna K. Ulatowska \\ Kristin King \\ Julene K. Johnson \\ Callier Center for Communication Disorders, \\ University of Texas at Dallas \\ Donald D. Mclntire \\ University of Texas Southwestern Medical Center, Dallas
}

The present study compared discourse ability across three groups: patients with mild Alzheimer's disease (AD), healthy oldelderly individuals (OE, $>\mathbf{8 0}$ years), and normal control subjects (NC). Discourse samples were analyzed according to aspects of coherence using a methodology based on frame analysis (Goffman, 1974). The results revealed significant differences in coherence between the AD and both the $O E$ and NC groups, on aspects of content as reflected in frame of interpretation and proportion of frame-supporting information. Differences were also found on measures of textual form of the responses. No significant differences were found between $\mathrm{OE}$ and NC groups, implicating relative preservation on these particular discourse measures with advanced age. The disparity in discourse abilities between the $A D$ and $O E$ groups suggests that discourse differences in early $A D$ are qualitatively different from those of normal advanced aging Explanations for the difficulties in the $A D$ population are delineated.

L anguage and communicative deficits have been documented during all stages of Alzheimer's disease (AD) (Bayles \& Kaszniak, 1987; Ulatowska \& Chapman, 1991). Nonetheless, it is difficult to differentiate between the language changes during the early stages of $\mathrm{AD}$ and language changes associated with normal aging. The difficulty arises from the overlap in patterns of language change observed in these groups. For example, word finding problems, difficulty with reference, reduced information content, and a tendency to digress have been identified in both populations (Nicholas, Obler, Albert, \& Helm.
Estabrooks, 1985; Ulatowska et al., 1988). A critical issue raised in the literature is whether language differences between early $\mathrm{AD}$ and normal advanced aging are a matter of degree or whether qualitative differences exist (Tomoeda \& Bayles, 1993; Ulatowska \& Chapman, 1991). Qualitative discourse differences have been implicated in behaviors such as omitting central information and events that require inferencing, as well as difficulty organizing and sequencing story information (Chenery \& Murdoch, 1994). Other evidence has pointed to an early vulnerability of discourse coherence in AD (Chenery \& Murdoch, 1994; Cherney \& Canter, 1992; Ripich \& Terrell, 1988).

Discourse studies in AD populations have emphasized the importance of selecting appropriate discourse tasks, stimuli, and measures to tap early discourse changes. A number of methodologies that use pictorial stimuli to study discourse in adult neurogenic populations are reported in the literature (Chenery \& Murdoch, 1994; Nicholas \& Brookshire, 1993; Tomoeda \& Bayles, 1993; Ulatowska \& Chapman, 1991). Pictorial stimuli that require holistic integration of the depicted information are more likely to tap early qualitative changes in $\mathrm{AD}$ than are pictures that tend to elicit isolated, unrelated observations (Chenery \& Murdoch, 1994). Furthermore, discourse measures such as the amount of language and/or information may be insensitive to early pathologic changes in AD (Chenery \& Murdoch, 1994; Tomoeda \& Bayles, 1993). In contrast, aspects of discourse such as conciseness and coherence appear to be compromised early in the disease process, hence offering guidelines for differentiating the populations (Chenery \& Murdoch, 1994; Ripich \& Terrell, 1988; Tomoeda \& Bayles, 1993).

\section{Framework and Purpose}

The present study investigates whether discourse differences exist between early stage $\mathrm{AD}$ and normal advanced aging. To investigate whether discourse measures can be used to discriminate pathologic changes in early $\mathrm{AD}$ from changes due to normal aging, we characterize discourse coherence using a method of frame analysis derived from the work of Goffman (1974). Theoretical evidence suggests that people are able to extract a considerable amount of information after only brief exposures to complex pictures or scenes (Biederman, 1972; Goffman, 1974; Tannen, 1979). That is, people attend to the depicted participants and props in pictures, recognize the salient cues, and arrive at an interpretation almost instantaneously. The processing of complex scenes is assumed to be guided by application of internalized knowledge structures referred to as frames. Frames are established through experience and define a set of possible actions and roles for the participants. Furthermore, application of a frame allows conjecture about what may have preceded or followed the current event to build a coherent narrative.

The primary issue addressed herein was whether discourse coherence differs on aspects of content and form (Table 1). With regard to content, we defined coherent responses as containing a holistic integration of the information (i.e., frame) 
as well as information supporting the frame. The issues addressed included whether (a) frame of interpretation and (b) amount of semantically related versus disruptive information differed across groups. With regard to form, we examined the textual structure of the response (i.e., narrative, partial narrative, or nonnarrative) and the use of reference.

\section{Method}

\section{Subjects}

Discourse processing was examined in three groups, including individuals with early stage $\mathrm{AD}(M$ age $=67.5$, range $51-$
76), normal old-elderly ( $O E$ ) individuals (>80 years) ( $M$ age $=81.9$, range 80-92). and normal control (NC) subjects $(M$ age $=65.7$, range $47-78$ ) matched in age to the $\mathrm{AD}$ group. The three groups of twelve subjects each did not differ significantly on the variables of gender ( 8 female, 4 male in each group), education, and professional background. The diagnosis of probable AD was made by the University of Texas Southwestern Medical Clinic for Alzheimer's and Related Diseases based on neurologic, cognitive, and behavioral testing using National Institute of Neurological and Communicative DisordersADRDA criteria (McKhann et al., 1984). All persons with $\mathrm{AD}$ scored 19 or higher
TABLE 1. Discourse coherence domains and the descriptive statistics for each group.

\begin{tabular}{|c|c|c|c|c|}
\hline \multirow{2}{*}{ Coherence } & \multirow[t]{2}{*}{$\begin{array}{c}\text { Discourse Components } \\
\text { Variables }\end{array}$} & \multicolumn{3}{|c|}{ Results } \\
\hline & & $A D$ & OE & NC \\
\hline
\end{tabular}

\section{Aspects of Content}

Frames of Interpretation

$\begin{array}{lccc}\text { Typical } & 19 & 33 & 36 \\ \text { Atypical } & 13 & 3 & 0 \\ \text { Incorrect } & 0 & 0 & 0 \\ \text { None } & 4 & 0 & 0 \\ & 59 \% & 86 \% & 90 \% \\ \text { Propositions Supporting Frame } & 83(21 \%) & 153(29 \%) & 182(28 \%) \\ \text { Core } & & & \\ \text { Elaborative } & 80(21 \%) & 130(25 \%) & 161(24 \%) \\ \quad \text { Explicit } & 67(17 \%) & 171(32 \%) & 253(38 \%) \\ \quad \text { Implicit } & 41 \% & 14 \% & 10 \% \\ \text { Propositions Disrupting Frame } & 0(0 \%) & 0(0 \%) & 0(0 \%) \\ \text { Irrelevant } & 6(1.5 \%) & 0(0 \%) & 0(0 \%) \\ \text { Incorrect } & 27(6.9 \%) & 12(2.3 \%) & 13(2 \%) \\ \text { Asides } & 37(9.5 \%) & 34(6.4 \%) & 33(5 \%) \\ \text { Repeated } & 14(3.6 \%) & 1(.19 \%) & 6(.9 \%) \\ \text { Empty } & 20(5.1 \%) & 9(1.7 \%) & 6(.9 \%) \\ \text { Incomplete } & 24(6.2 \%) & 8(1.5 \%) & 4(.6 \%) \\ \text { Metacognitive } & 31(8.0 \%) & 10(1.9 \%) & 2(.3 \%) \\ \text { Atypical } & & & \end{array}$

\section{Aspects of Form}

Structure of Information

\begin{tabular}{lccc} 
Narrative & $4(11 \%)$ & $26(72 \%)$ & $32(89 \%)$ \\
Partial narrative & $24(67 \%)$ & $9(25 \%)$ & $4(11 \%)$ \\
Nonnarrative & $8(22 \%)$ & $1(3 \%)$ & $0(0 \%)$ \\
Reference & & & \\
Pronoun/referent ratio & 1.50 & 1.89 & 1.98 \\
Mean referential errors & 5.66 & 5.83 & 2.80 \\
\hline
\end{tabular}

Note. Integers represent total number of responses for each variable collapsed across stories (exception: figures for reference). Percentages represent the proportion of response category type per total responses produced for each group collapsed across stories. on the Mini-Mental State Examination (MMSE: Folstein. Folstein. \& McHugh. 1975) $(M$ score $=22.4$, range $19-29, S D=$ 3.3). Individuals were excluded if they had a history of cardiovascular disease. diabetes mellitus, cerebrovascular accident, epilepsy, serious head injury, or alcohol abuse, or a positive psychiatric history,

The standardized language and cognitive measures administered to all subjects are shown in Table 2. As indicated, the OE and NC groups did not differ significantly on any of the measures. In contrast, the AD group performed significantly lower than either normal group on all but two measures.

\section{Procedures}

\section{Stimuli}

The picture stimuli consisted of three Norman Rockwell prints. Rockwell pictures were chosen because they are contextually rich, representing a salient era of the subjects' lives; require a holistic integration of depicted information; and are widely used for research and clinical applications (Myers, 1991; Tomoeda \& Bayles, 1993; Ulatowska \& Chapman, 1991). Each pictorial stimulus illustrated a familiar scene of a well-known life situation. The situations included: a son going to college, a boy running away from home, and a soldier returning home from war.

\section{Testing}

The subjects were tested individually in a quiet room. They were allowed to view the picture stimulus for as long as desired. The picture was then turned face down to reduce the tendency to produce a picture description. However, the subjects could review the picture. In a method similar to that described by Chenery and Murdoch (1994), the subjects were allowed to view the picture while producing a story if their short-term memory deficits precluded relating information about the picture. The subjects were asked to create a story about the picture. They were instructed not to describe the picture but to generate a possible sequence of events. All responses were audiotaped and transcribed verbatim by the examiner. A second individual listened to and edited the transcripts to ensure transcript accuracy.

\section{Discourse Analysis}

The discourse responses were analyzed according to aspects of content and 
TABLE 2. Comparison of performance across groups on standardized language and cognitive tests.

\begin{tabular}{|c|c|c|c|}
\hline & $\begin{array}{c}A D \\
\text { Mean }(S D)\end{array}$ & $\begin{array}{c}\mathrm{OE} \\
\text { Mean }(S D)\end{array}$ & $\begin{array}{c}\mathrm{NC} \\
\text { Mean }(S D)\end{array}$ \\
\hline \multicolumn{4}{|l|}{ I. Standardized language tests: } \\
\hline \multicolumn{4}{|l|}{ Boston Diagnostic Aphasia Examination } \\
\hline Complex ideational $(12)^{\star}$ & $8.3(3.0)^{a}$ & $10.9(1.1)^{b}$ & $11.1(0.7)^{\mathrm{b}}$ \\
\hline $\begin{array}{l}\text { Reading Sentences and Paragraphs } \\
(10)^{\star} \text { (Goodglass \& Kaplan, 1983) }\end{array}$ & $8.9(1.4)^{a}$ & $9.3(0.8)^{\mathrm{ab}}$ & $9.9(0.3)^{b}$ \\
\hline \multicolumn{4}{|l|}{ Modified Boston Naming Test } \\
\hline $\begin{array}{l}(30)^{\star} \text { (Kaplan, Goodglass, \& Weintraub, } \\
1983)\end{array}$ & $18.8(8.9)^{\mathrm{a}}$ & $26.1(2.3)^{\mathrm{b}}$ & $28.1(1.8)^{\mathrm{b}}$ \\
\hline \multicolumn{4}{|l|}{ II. Standardized cognitive tests: } \\
\hline $\begin{array}{l}\text { Mini-Mental State Examination }(30)^{*} \\
\text { (Folstein, Folstein, and McHugh, 1975) }\end{array}$ & $22.4(3.3)^{a}$ & $29.0(0.6)^{b}$ & $29.0(0.6)^{\mathrm{b}}$ \\
\hline $\begin{array}{l}\text { Raven's Coloured Progressive Matrices } \\
(24)^{\star} \text { (Raven, 1962) }\end{array}$ & $16.0(5.3)^{\mathrm{a}}$ & $17.9(2.9)^{\mathrm{a}}$ & $21.1(3.2)^{\mathrm{a}}$ \\
\hline \multicolumn{4}{|l|}{ Wechsler Adult Intelligence Scale-R } \\
\hline Picture Arrangement $(10)^{\star \star}$ & $5.4(3.1)^{\mathrm{a}}$ & $11.0(3.1)^{\mathrm{b}}$ & $12.9(2.4)^{b}$ \\
\hline Block Design $(10)^{* *}$ & $6.4(2.6)^{\mathrm{a}}$ & $10.8(3.0)^{b}$ & $13.0(2.9)^{\mathrm{b}}$ \\
\hline Similarities $(10)^{\star \star}$ Wechsler, (1981) & $7.9(3.6)^{\mathrm{a}}$ & $13.3(1.4)^{b}$ & $12.7(2.3)^{\mathrm{b}}$ \\
\hline $\begin{array}{l}\text { Wechsler Logical Memory Scale-R }(50)^{*} \\
\text { (Wechsler, 1987) }\end{array}$ & $3.7(2.2)^{\mathrm{a}}$ & $23.0(7.1)^{b}$ & $29.1(7.9)^{\mathrm{b}}$ \\
\hline
\end{tabular}

Note. Populations without same superscript differed significantly $(p<0.05)$

*Maximum possible score

**Mean scaled score

form related to coherence (Table 1). To address content aspects of coherence, the frame of interpretation and the relation of the individual propositions to the frame were analyzed. Each discourse response was classified as representing a typical, atypical, incorrect frame, or no frame. The typical frames were derived a priori from the title given by the artist. The typicality of frames was validated by a normal group $(N=40$, age range 45-75) who produced typical frames $100 \%$ of the time. The typical frames for the three stimulus pictures were: a son going to college, a boy running away from home, and a soldier returning home from war. These are subsequently referred to as "College," "Runaway," and "Soldier" frames. Atypical frames were those in which the subject failed to decipher the salient relationships between the characters and props and/or failed to holistically integrate all the salient information related to the depicted situation. For example, an atypical frame for the "College" picture may involve a failure to interpret the "father/son" relationship or a failure to realize that the son was leaving for college (see Appendix A for examples). An incorrect frame was one that was not recoverable from the depicted information. Responses coded as "no frame" were manifested by a failure to integrate the relationships and events into any schema at all.

To analyze the relationship of the individual propositions to the frame of interpretation, the discourse samples were first segmented into propositions consisting of a verbal element and its modifying arguments. The propositions were then classified according to variables in the categories of frame-supporting propositions or frame-disrupting propositions (Table 1). Frame-supporting propositions encompassed core or elaborative information. The core propositions consisted of information that supported the typical frame and were mentioned by over $75 \%$ of the normal group. Elaborative propositions were semantically related to the typical frame conveying information beyond the core information. In addition, elaborative propositions were coded as either explicit (depicted in the picture) or implicit (required inferencing). In contrast, propositions disrupting the frame consisted of the following types of information: (a) irrelevant-information unrelated to frame; (b) incorrect-information inconsistent with picture stimuli; (c) asidepersonal comments and digressions; (d) repeated-redundant information; (e) empty-information lacking enough specificity to contribute content; (f) incomplete-information left dangling; $(\mathrm{g})$ metacognitive-comments reflecting on performance, for example: "I can't remember," "I used to be good at this"; and $(h)$ atypical-information that was plausible but did not support the typical frame.

To address differences in the form of discourse, the discourse responses were classified according to the type of narrative. The stories were coded as a narrative (i.e., setting, action, and closing), partial narrative (i.e., omission of a story component or interruption of the story), or nonnarrative (description of pictured information). In addition, referential analysis was performed to evaluate strategies for using references (pronounto-referent ratio for the depicted persons) and to assess errors of reference leading to ambiguity. Referential errors included pronouns where no clear referent was recoverable or when more than one referent could reasonably be assigned to a pronoun. Reference is an important cohesive device because of its impact on coherence of narratives.

\section{Reliability}

Discourse analyses were conducted independently by two trained coders for all discourse samples for frame and narrative organization and for one-third of the discourse samples for frame-supporting propositions, frame-disrupting propositions, and errors of reference. Point-bypoint reliability was calculated by dividing the number of agreements by the number of agreements plus disagreements and multiplying by 100 . Reliability was $97 \%$ for frame, $95 \%$ for narrative organization, $92 \%$ for frame-supporting propositions, $92 \%$ for frame-disrupting information, and $90 \%$ for errors of reference.

\section{Statistical Methods}

For categorical data, such as "Differences in the Frame of Interpretation," the group percentages were compared with the chi-square statistic. For some responses, small cell sizes warranted the use of exact tests rather than chi-square. In these cases, only the $p$-value is reported. For statistically significant results, the comparisons were partitioned to determine the pairwise significance. Bonferroni corrections were used for these comparisons. For the continuous measures, such as "Proportion of Pronouns to Total Referents," the groups were compared using one-way. analysis of variance (ANOVA). The statistics reported for the ANOVAs are $F$-statistics. Pairwise comparisons were 
made for significant analyses of variance with the Tukey-Kramer HSD multiple comparison. Statistical significance was accepted for any tests with a $p$-value less than 0.05. Descriptive statistics are reported in Table 1.

\section{Results}

\section{Content Aspects of Coherence}

Frame of Interpretation. For each picture, the frame of interpretation (i.e., typical, atypical, incorrect, or no frame) was examined for differences in proportions across groups (see Appendix for examples). Figure 1 shows the pattern of results across frame types. The NC and OE groups used a typical frame of interpretation in most instances. In contrast, the mild $\mathrm{AD}$ patients applied a typical frame only $50 \%$ of the time. They often applied atypical frames or failed to interpret the picture within any frame. The differences between the patients with mild AD and the $\mathrm{OE}$ and NC groups were significant for the "College" frame $\left(\mathrm{X}^{2}(4)=10.71, p=\right.$ $0.030)$ and the "Runaway" frame $\left(\mathrm{X}^{2}(4)=\right.$ $13.75, p=0.0081$ ).

Frame-Supporting Information. The frame supporting variable included core and elaborative propositions. The mild AD group produced significantly fewer core and elaborative propositions as compared to the $\mathrm{OE}$ and $\mathrm{NC}$ groups, $F(2,33)=7.53$; $p=0.002$, who did not differ significantly from one another. Figure 2 shows the percentage of core propositions produced out of the total set of possible core propositions collapsed across stories.

The differences between groups in type of elaborative information, i.e., explicit versus implicit, failed to reach significance, $F(2,33)=3.12 ; p=0.0573$. Nonetheless, a trend in the expected direction was found (AD > OE and NC on explicit).

Frame-Disrupting Information. The informational units classified as frame disrupting (Table 1) were collapsed across types for group comparisons. The results revealed that the mild $A D$ group used significantly more frame disrupting information than the OE or NC groups, $F(2,33)=3.97 ; p=0.0285$.

Frame-Supporting Versus FrameDisrupting Information. To compare the proportion of propositions that supported the frame, the number of frame-supporting propositions was divided by the total propositions produced and compared across groups. The mild $\mathrm{AD}$ group used a significantly smaller proportion of framesupporting propositions as compared to the $\mathrm{OE}$ and NC groups, $F(2.33)=21.097$; $p=0.0001$ (Figure 3). The OE and NC groups did not differ significantly.

FIGURE 1. Percentage of frame interpretation per category collapsed across three discourse tasks.

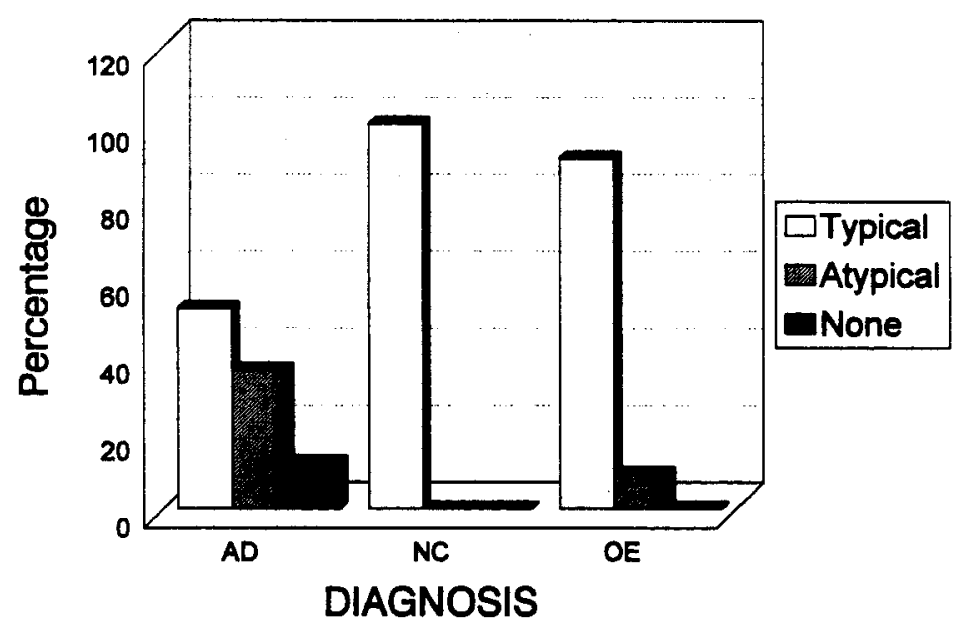

FIGURE 2. Percentage of frame supporting core information collapsed across three discourse tasks.

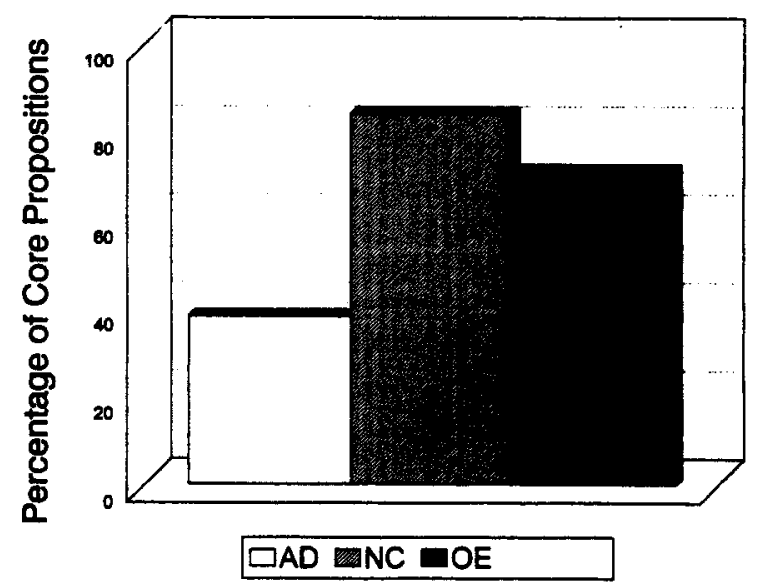

\section{Aspects of Form}

Structure of Information. The subjects' discourse responses for each picture were coded according to how the information was organized (i.e., narrative, partial narrative, or nonnarrative). The mild $\mathrm{AD}$ subjects provided significantly fewer narrative responses than the $\mathrm{OE}$ or the $\mathrm{NC}$ groups across all pictures (exact test values: $p=0.0004$, "College" frame; $p=$ 0.0002 , "Runaway" frame; and $p=$ 0.0001 , "Soldier" frame). (See Figure 4.)

\section{Use of Reference}

Unexpectedly, the proportion of pronouns to total referents, $F(2,33)=1.98$, $p=0.1539$, and the number of errors,
$F(2.33)=1.23 ; p=0.3041$, did not differ significantly across groups. The mean number of referential errors was relatively higher in both the $\mathrm{AD}(M=5.66, S D=$ 3.96) and the OE group $(M=5.83, S D=$ 7.66) compared to the NC group $(M=2.8$, $S D=2.92$ ). There was, however, considerable variability within each group, particularly the OE group.

\section{Discussion}

The present study extends previous work demonstrating impaired discourse processing in patients with AD (Chenery \& Murdoch, 1994; Tomoeda \& Bayles, 1993; Ulatowska et al., 1988; Ulatowska \& Chapman, 1991) by considering whether language decline in early $\mathrm{AD}$ is 
FIGURE 3. Proportion of frame supporting propositions (core and elaborative) to total propositions (frame supporting and frame disrupting) collapsed across three discourse tasks.

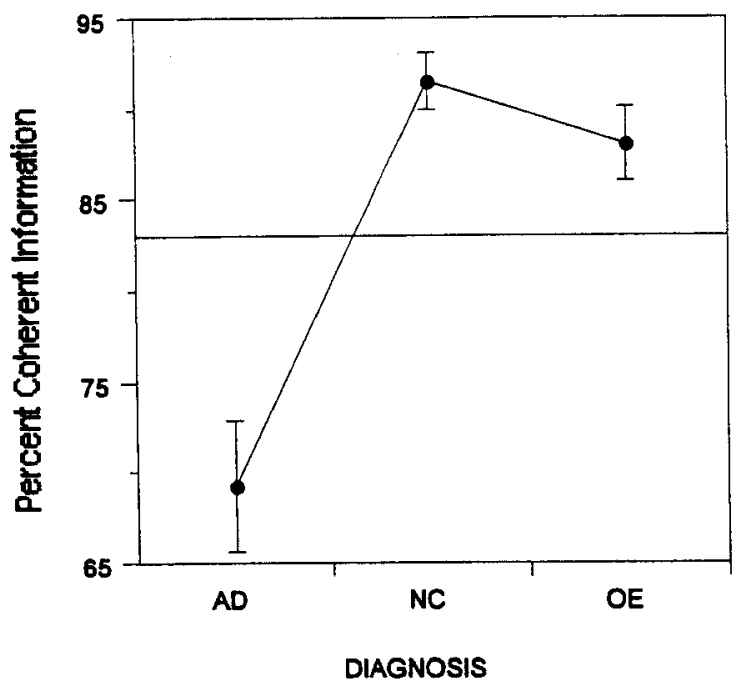

FIGURE 4. Percentage of responses classified according to structure of information collapsed across three discourse tasks.

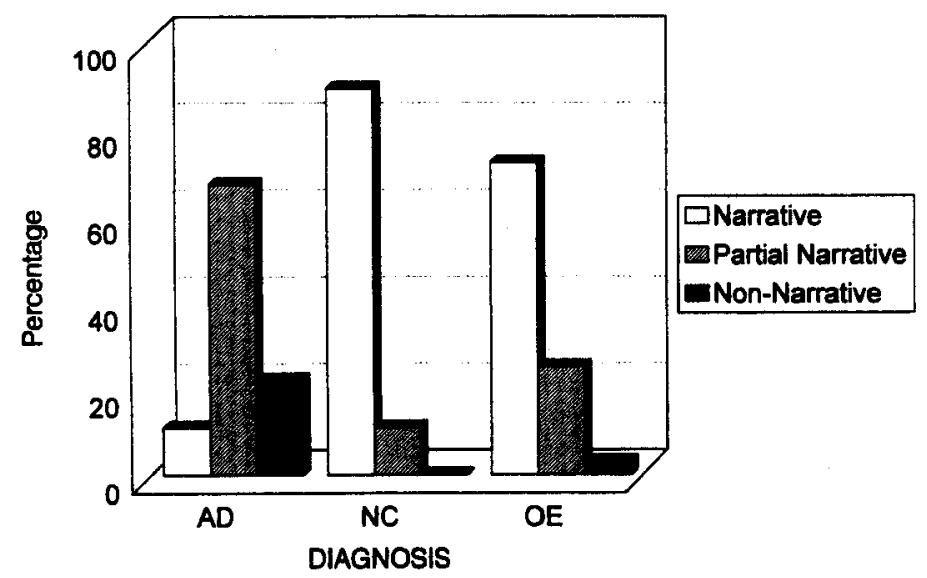

qualitatively different from changes observed in normal advanced aging. The patients with early AD were impaired significantly compared to $\mathrm{OE}$ and $\mathrm{VC}$ subjects on a range of discourse coherence measures that included application of a typical frame of interpretation and generation of frame-supporting information.

The difficulty in applying a typical frame of reference in mild AD may arise from a variety of underlying factors such as memory deficits, attentional deficits, visual perceptual problems, disruption of internalized frame representation, or failure to access frame knowledge. If little or no trace of the depicted information remains in the subject's working memory, the subject will have difficulty integrating the stimulus information with stored knowledge of frames when the picture is removed. In addition, ability to apply a typical frame may be compromised by attentional deficits manifested in a failure to attend to the salient props. It is also possible that visual perceptual problems may add to the difficulty observed in early AD patients in integrating the depicted information in a coherent frame. Certain subjects also may fail to produce a typical frame as a result of breakdown in the internalized frame representation.

The patients with mild AD also produced fewer responses in the form of narratives as their narrative attempts were partial narratives (either narrative fragments or narratives interrupted by frame disrupting responses) or picture descriptions (nonnarratives). In contrast, the $\mathrm{OE}$ and NC groups had little difficulty transforming the information depicted in a static picture to a dynamic, narrative form.

The failure to access a frame may correspond to an inability to generate a response in narrative form. The patients with $\mathrm{AD}$ who failed to interpret a picture within a particular frame were also unsuccessful at producing a response in narrative form. Thus, accessing a frame may be prerequisite to conjecturing about preceding or subsequent events to guide coherent narrative production from a static picture. However, producing a typical frame interpretation does not necessarily ensure ability to formulate a narrative. For example, even though the mild $\mathrm{AD}$ group exhibited more typical frame interpretations on the "Soldier" frame, they produced fewer narrative and partial narrative responses and less frame-supporting core information than the two normal groups.

Even though the present results indicate that discourse coherence is impaired in early $A D$, our results must be interpreted cautiously due to the small sample size. Moreover, our group of OE may represent an elite group of successful aging, rather than normal aging, individuals. Additionally, the results were not universally consistent across each $A D$ patient. Two patients with mild AD were not clearly distinguishable from our $\mathrm{OE}$ group. However, these two patients overlapped two $O E$ individuals who produced the least amount of framesupporting core information $(50 \%)$. The possibility exists that the two patients with AD may have had high premorbid IQ scores or that the two OE may be at risk for developing dementia.

The ability to interpret a frame, to supply frame supporting information, and to integrate the information in narrative form appears to be relatively preserved in normal advanced aging. Moreover, the findings suggest that these same discourse parameters are impaired in early AD. The data support the clinical utility of discourse measures to distinguish between early changes in $\mathrm{AD}$ and changes due to normal advanced aging. That is, a methodology that requires holistic integration of visual information to create a narrative may be valuable in tapping the early discourse changes in $A D$. Whether or not the differences in $A D$ will be distinguishable from discourse in 
other neurogenic populations with either left or right hemispheric stroke warrants investigation.

\section{Acknowledgments}

This investigation was supported by grants from the National Institute of Aging/National Institutes of Health (AG09486 and 5-P30-AG12300-02). We thank Angela Shobe for her arduous work in preparing this manuscript. We also acknowledge with gratitude our patients with Alzheimer's disease for teaching us how to interact with them as people beyond a disease and our healthy oldelderly individuals for showing us how to live.

\section{References}

Bayles, K. A., \& Kaszniak, A. W. (1987). Communication and cognition in normal aging and dementia. Boston: Little, Brown.

Biederman, I. (1972). Perceiving real-world scenes. Science, 177, 77-80.

Chenery, H. J., \& Murdoch, B. E. (1994). The production of narrative discourse in response to animations in persons with dementia of the Alzheimer's type: Preliminary findings. Aphasiology, 8, 159-171.

Cherney, L. R., \& Canter, G. J. (1992). Informational content in the discourse of patients with probable Alzheimer's disease and patients with right brain damage.
Clinical Aphasiology: 21, 123-134.

Folstein, M. F., Folstein, S. E., \& McHugh, P. R. (1975). "Mini-Mental State": A practical method for grading the mental state of patients for the clinician. Journal of Psichi. atric Research, 12, 189-198.

Goffman, E. (1974). Frame analvsis: An essar on the organization of experience. Cambridge, MA: Harvard University Press.

Goodglass, H., \& Kaplan, E. (1983). The assessment of aphasia and related disorders. Philadelphia, PA: Lea \& Febiger.

Kaplan, E., Goodglass, H., \& Weintraub, S. (1983). Boston Naming Test. Philadelphia: Lea \& Febiger.

McKhann, G., Drachman, D., Folstein, M., Katzman, R., Price, D., \& Stadlan, E. M. (1984). Clinical diagnosis of Alzheimer's disease. Neurology, 34, 939-944.

Myers, P. S. (1991). Inference failure: The underlying impairment in right-hemisphere communication disorders. Clinical aphasiology, 20, 167-180. Austin: Pro-Ed.

Nicholas, L. E., \& Brookshire, R. H. (1993). A system for quantifying the informativeness and efficiency of the connected speech of adults with aphasia. Journal of Speech and Hearing Research, 36, 338-350.

Nicholas, M., Obler, L., Albert, M., \& HelmEstabrooks, N. (1985). Empty speech in Alzheimer's disease and fluent aphasia. Journal of Speech and Hearing Research, 28, 405-410.

Raven, J. (1962). Coloured progressive matrices. London: H. K. Lewis.

Ripich, D. N., \& Terrell, B. Y. (1988). Patterns of discourse cohesion and coherence in
Alzheimer"s disease. Journal of Speech and Hearing Disorders. 53. 8-15.

Tannen, D. (1979). What 's in a frame? In R. O Freedle (Ed.). Neli directions in discourse processing (pp. 137-181). Norwood. NJ: Ablex Publishing.

Tomoeda, C. K., \& Bayles, K. A. (1993). Longitudinal effects of Alzheimer disease on discourse production. Alzheimer Disease and Associated Disorders. 7, 223-236.

Ulatowska, H. K., Allard, L., Donnell, A., Bristow, J., Haynes, S. M., Flower, A., \& North, A. J. (1988). Discourse performance in subjects with dementia of the Alzheimer" type. In H. Whitaker (Ed.), Neuropsychological studies in nonfocal brain damage (Vol. 2 , pp. 108-131). New York: Springer-Verlag.

Ulatowska, H. K., \& Chapman, S. B. (1991). Discourse studies. In R. Lubinski (Ed.), Dementia and communication (pp. 115-132). Philadelphia: B. D. Decker.

Wechsler, D. (1981). Wechsler Adult Intelligence Scale-Revised. New York: Psychological Corporation.

Wechsler, D. (1987). Wechsler Memorv ScaleRevised. San Antonio, TX: Psychological Corporation.

Contact author: Sandra Bond Chapman, $\mathrm{PhD}$, Callier Center for Communication Disorders, University of Texas at Dallas, 1966 Inwood Road, Dallas, TX 75235

Key Words: discourse, Alzheimer's disease, normal aging, coherence, language

\section{Appendix A}

\section{Samples of Frame of Interpretation for "College" Frame}

\section{Typical Frame (NC)}

Well, young Tom had just uh graduated from high school and was gettin' ready to go off to uh the State University uh probably his first time away from home. He was a farm boy, and uh I guess he was headed for the big city. He uh he's very anxious uh, looking around and waiting for the train I presume. And uh his dog realizes that uh something's up. He-he realizes the little boy's gonna go away, and he's got his head on the boy's knee. And the father's sittin' there uh realizing that the boy is gonna leave home for the first time and he's kind of dejected. He's uh doesn't want to see him go, but on the other hand, he realizes he's a big boy now, and he's going off into the world.

\section{Typical Frame (AD) (Notice uncertainty in expression)}

Well uh the way I interpret that that your uh is that-uh a father and a son uh are waiting for a bus or for some choc- some type of transportation to take them somewhere, and um they're just un that's about all I can think of uh- (Examiner: Where they are going?)...Well, the boy is dressed up, and the and the father isn't, so maybe he is taking him to school. Un that would be my one guess. I don't know what the dog's doing there, but uh I would the dog wouldn't necessarily come along uh for that purpose, so maybe that's not the correct answer.

\section{Atypical Frame (AD)}

Okay, there's an older man and a young boy and a dog. And it-and it looks like a big long truck there that I can see. It's a plain yard and, uh, one of em's looking one way, and one's looking the other, and the dog's just got his neck on there and just sitting there. I don't know, it must be their dog, but some dogs do that anyway whether you do or not. So they might be having trouble with that - with that truck, or that - that older man might be. I don't know if that young one could drive one, drive one that far. So evidently they're sittin' there, they might be having trouble, but looks like they're sittin' there quite a while. And one of 'em was loo-the man was looking one way, and that - that young guy was looking another way, and then the dog was just sittin' there with his head on it looking.

\section{No Frame (AD)}

Now that's a little bit older boy there looks like. It is a boy isn't it? Well, this is his father. And there he's smoking a cigarette. I don't agree on that. That's one thing in my family. No smoking. I've never smoked in my life. Now he's looking at what his father's doing. I believe that's the dog. 